\title{
iFRET: An Improved Fluorescence System for DNA-Melting Analysis
}

\author{
W. Mathias Howell, ${ }^{1}$ Magnus Jobs, and Anthony J. Brookes \\ Center for Genomics and Bioinformatics, Karolinska Institute, S-171 77, Stockholm, Sweden
}

\begin{abstract}
Fluorescence resonance energy transfer (FRET) is a powerful tool for detecting spatial relationships between macromolecules, one use of which is the tracking of DNA hybridization status. The process involves measuring changes in fluorescence as FRET donor and acceptor moieties are brought closer together or moved farther apart as a result of DNA hybridization/denaturation. In the present study, we introduce a new version of FRET, which we term induced FRET (iFRET), that is ideally suited for melting curve analysis. The innovation entails using a double-strand, DNA-specific intercalating dye (e.g., SYBR Green I) as the FRET donor, with a conventional FRET acceptor affixed to one of the DNA molecules. The SNP genotyping technique dynamic allele specific hybridization (DASH) was used as a platform to compare iFRET to two alternative fluorescence strategies, namely, the use of the intercalating dye alone and the use of a standard FRET pair (fluorescein as donor, 6-rhodamine as acceptor). The iFRET configuration combines the advantages of intercalating dyes, such as high signal strengths and low cost, with maintaining the specificity and multiplex potential afforded by traditional FRET detection systems. Consequently, iFRET represents a fresh and attractive schema for monitoring interactions between DNA molecules.
\end{abstract}

Fluorescence signals may be created by various means to detect DNA hybridization during genotyping and similar assays. The simplest method is to use an intercalating dye (Fig. 1a) that is highly specific for double-stranded DNA. When such a dye (e.g., SYBR Green I) intercalates into a DNA double helix, the dye can fluoresce while exposed to suitable excitatory illumination. Subsequent separation of the DNA strands as part of an assay procedure will cause a release of the dye and consequential loss of fluorescence. This strategy is very inexpensive and yields high levels of fluorescence (Howell et al. 1999), but its inherent limitations include a lack of specificity for any particular duplex and no possibility to create multiplexed assays.

A second strategy for the detection of DNA hybridization involves fluorescence resonance energy transfer, or FRET (Fig. 1b). In FRET, a donor fluorophore molecule absorbs excitation energy and delivers this via dipole-dipole interaction to a nearby acceptor fluorophore molecule (Stryer and Haugland 1967), as recently reviewed (Wu and Brand 1994). This process only occurs when the donor and acceptor molecules are sufficiently close to one another. Several different strategies for determining the optimal physical arrangement of the donor and acceptor moieties have been described (Holland et al. 1991; Tyagi and Kramer 1996; Bernard et al. 1998; for review, see Didenko 2001; Solinas et al. 2001). Although FRET enables specification of the target sequence and the potential for multiplexing, this is counterbalanced by the extra expense of physically attaching both the donor and acceptor fluorophores, and a much weaker relative fluorescence intensity.

\section{'Corresponding author.}

E-MAIL Mathias.Howell@cgb.ki.se; FAX 46 (0)8-324826. Article and publication are at http://www.genome.org/cgi/doi/10.1101/ gr.297202.
Here, we present an alternative way to create fluorescence signals, via a merger of the above-mentioned strategies. We have found that SYBR Green I acts as a very effective FRET donor reagent for several different acceptor molecules (Fig. 1c) and thus may be used in place of the usual bound donor molecule. Although a similar combination of nonattached FRET donor dye plus attached FRET acceptor molecule has been described as a means to observe DNA (Cardullo et al. 1988), we show here that using a highly double-strand specific dye (e.g., SYBR Green I) provides a multitude of advantages, including very high signal strength, increased specificity, and much reduced background. This is because the donor signal that is "induced" by the dye intercalation is both relocated away from the acceptor and simultaneously extinguished upon DNA denaturation. Hence, the use of an intercalating dye as a donor for tracking DNA denaturation is a concept we term induced FRET (iFRET).

In this paper, we compare and contrast the above three alternative fluorescent strategies to detect DNA hybridization status. As a platform for this, we use the SNP-genotyping method called dynamic allele-specific hybridization (DASH) (Howell et al. 1999). Beyond testing in model systems, the three fluorescence systems were validated on PCR-generated targets. Issues such as fluorescence signal intensity, singlebase allele discrimination, and alleviation of background fluorescence are explored.

\section{RESULTS}

The basic goal of many fluorescence procedures is to produce signals indicative of the presence of double-stranded DNA, wherein these signals change or disappear when the DNA becomes single stranded. In our study we have explored a new means for creating such signals by a reaction concept we call induced FRET (iFRET), as illustrated in Figure 1c. The experimental strategy we used was to evaluate the relative merits of iFRET and two commonly used alternatives, namely, interca- 


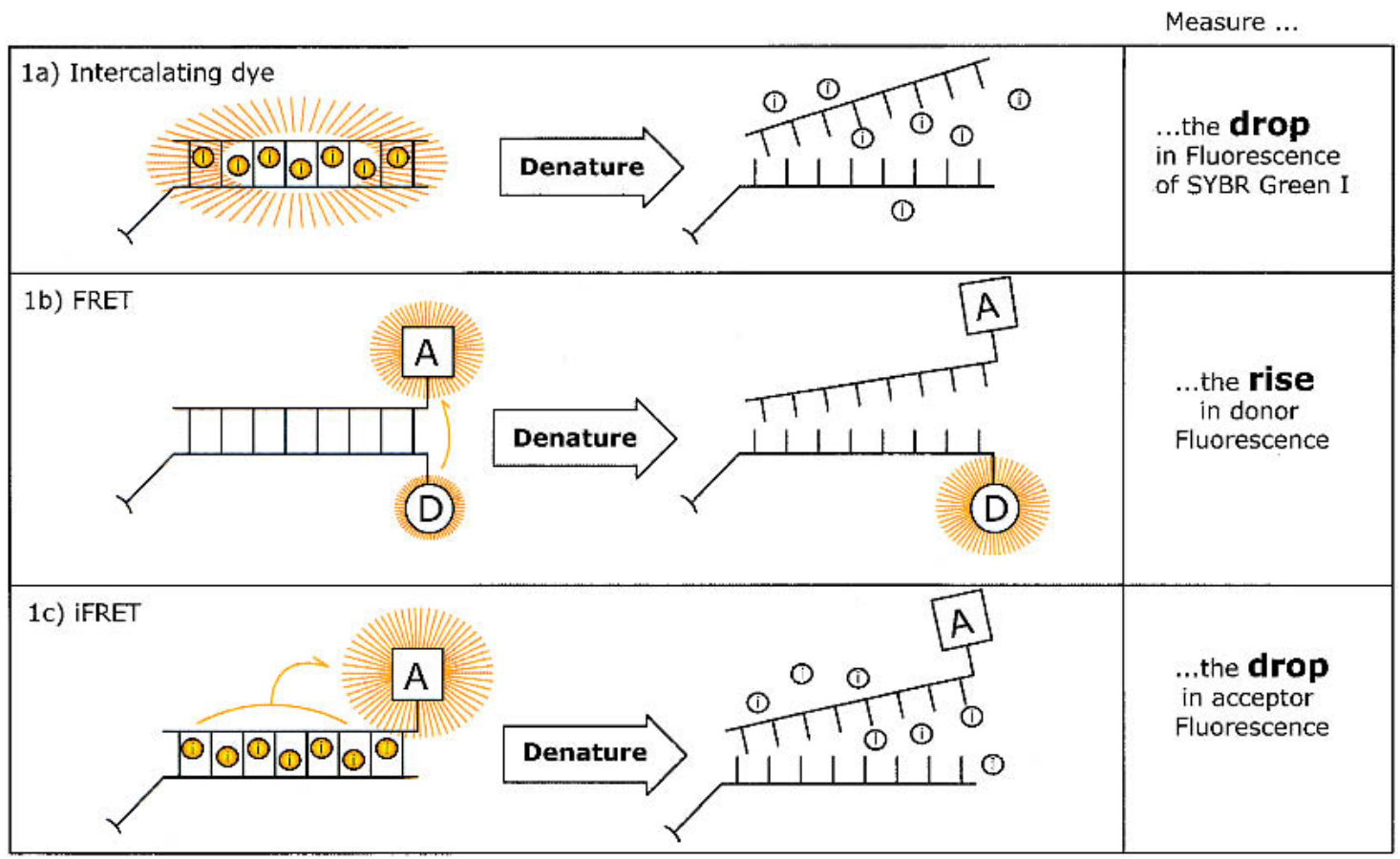

Figure 1 Three strategies for detecting DNA hybridization with fluorescence. Fluorescent outputs are indicated by radiating lines. (i) Intercalating dye, $(D)$ donor moiety, and $(A)$ acceptor moiety.

lation of a double-strand-specific DNA dye (Fig. 1a), and the use of standard FRET chemistry (Fig. 1b).

\section{Assay System Refinements}

\section{Dye Selection}

For each fluorescence platform, an appropriate dye or combination of dyes had to be selected. For use in intercalating dye detection, SYBR Green I was chosen because of its high specificity for double-stranded DNA and its bright fluorescence. For FRET, a series of donor molecules including FAM, JOE, Alexa 488, Bodipy FL, and Bodipy 530/560 were tested in combination with a number of acceptor molecules including ROX, TAMRA, Bodipy TMR, Bodipy 576/589, Bodipy TR, and even the "dark quencher" DABCYL (data not shown). Full descriptions of these dyes can be found at the Molecular Probes website (http://www.probes.com). We settled on the classic FRET combination of FAM donor and ROX acceptor for further experimentation. The main reasons for selecting this FRET pair were that (1) FAM is efficiently excited by 488-nm light emitted by the Argon laser (the light source in the ABI $7700)$, and (2) the emission maximum of FAM (540 nm) was easy to separate from the emission of $\operatorname{ROX}(611 \mathrm{~nm})$. For iFRET, a number of the aforementioned FRET acceptor molecules work well in combination with SYBR Green I as the donor. For our investigations, ROX was chosen to allow more direct comparison between FRET and iFRET results.

\section{FRET-Pair Location}

Aside from dye selection, another important issue was to determine the optimal spatial arrangement of the acceptor and donor molecules along the DNA duplex to optimize FRET. To examine this, a PCR-amplified target bearing a FAM (donor) molecule was used in combination with a sequential set of
3 '-ROX (acceptor)-labeled probes. Figure 2 summarizes the series of probe sequences and the relative locations of the donor and acceptor positions. Over this range of 10 bases, the precise relative position of the donor and acceptor was found to make little or no difference to created FRET signals. The experiment was repeated, with the ROX on the target and FAM on the probes, thus reversing the donor and acceptor locations. Again, different relative fluorophore positions had little or no effect on the generated FRET signals. When measuring donor fluorescence in these experiments, we observed that the donor-target plus acceptor-probe combination produced consistently stronger signals compared with the acceptor-target plus donor-probe alternative. The former arrangement was therefore used in subsequent investigations presented below.

\section{(D)}

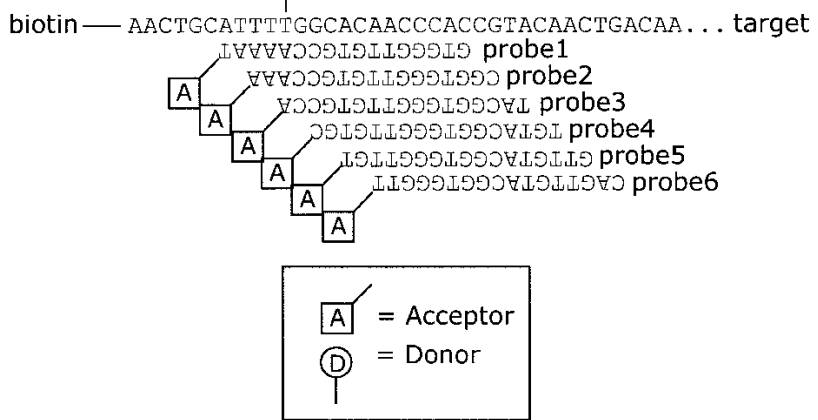

Figure 2 Probe series and target for fluorescence resonance energy transfer (FRET) pair testing. 


\section{Model System Experiments}

To compare the iFRET, FRET, and intercalating dye formats under controlled conditions, a model system based on synthetic oligonucleotides was used. A 17-bp oligonucleotide (5'-biotin-AACCCACCGTACAACTG) was used as a common immobilized target molecule. For detection using SYBR Green I, the hybridization solution included a $1 \times$ concentrate of the dye (diluted from the $10,000 \times$ concentrate stock provided by the manufacturer) along with an unlabeled complementary probe (5'-CAGTTGTACGGTGGGTT). To examine FRET, a fluorescence donor (FAM) was attached to the 3 '-end of the immobilized target, and an acceptor (ROX) was added to the 5 '-end of the probe. The same 5'-ROX-labeled probe was used for the iFRET format, wherein SYBR Green I dye (in a 1:10,000 dilution) was added in place of the FAM donor.

\section{Signal-Intensity Trends}

We observed a dramatic difference in fluorescence intensity between the three detection schemes (see Fig. 3). The difference between maximum and minimum fluorescence values was 5950 relative fluorescence units (RFUs) for iFRET, 2360 RFU for SYBR Green I, and 140 RFU for FRET. Thus, the iFRET gave fluorescence values that were 2.5 times greater than those obtained from the intercalating dye alone, and $>40$ times greater than those from FRET. The reason for this difference may be that the iFRET system involves the channeling of an accumulation of energy from a chain of donor dye molecules (in contrast to a single donor in the FRET system) into the acceptor moiety, which is then able to emit this energy unhindered. The same starting energy in the SYBR Green I system is presumably hindered from such efficient emission by effects such as shielding and energy reuptake by the many surrounding SYBR Green I molecules. Also, differing values for quantum yield and extinction coefficients may well contribute to the difference in emission intensity observed between iFRET and SYBR Green I.

\section{Allele Discrimination}

To examine the suitability of iFRET and the other systems for allele discrimination, a second oligonucleotide target (5'-biotinA A C C CACCATACAACT G ; underlined base indicating a 1-nt difference from the original target) was introduced into the three detection schemes. Hybridization of the above probes to this new target produces a single base mismatch in the center of the probe/target duplex. When run in parallel, these combinations equate to scoring two alternative homozygous genotypes for a naturally occurring singlenucleotide polymorphism.

Fluorescence versus temperature data for the matched and mismatched duplexes are presented in Figure 4. The derivative of the fluorescence as a function of temperature is shown beside the primary fluorescence curves. To have all derivative graphs depict comparable upward-facing peaks, the negative derivatives for the intercalating dye and iFRET values are given, but the positive derivative is given for FRET.

As disparate as the absolute fluorescence signal intensities were, all three detection systems were more than sufficient to enable facile allele discrimination (Fig. 4). The $T_{\mathrm{m}}$ differences between match and mismatch duplexes were readily derived from the observed peak temperatures. FRET gave the largest difference, with a mismatch peak at $49.7^{\circ} \mathrm{C}$ and a match peak at $60.8^{\circ} \mathrm{C}$, resulting in an $11.1^{\circ} \mathrm{C}$ separation between match and mismatch peaks. Use of SYBR Green I alone gave a mismatch peak at $64.3^{\circ} \mathrm{C}$ and a match peak at $72^{\circ} \mathrm{C}$, resulting in a difference in $T_{\mathrm{m}}$ of $8.3^{\circ} \mathrm{C}$. For iFRET, the values were $65.8^{\circ} \mathrm{C}, 72.9^{\circ} \mathrm{C}$, and $7.1^{\circ} \mathrm{C}$, respectively. The smaller window of peak separation noted for the SYBR Green I and iFRET schemes compared with the FRET alternative is presumably owing to the presence of the SYBR Green I dye. This dye is known to have a stabilizing effect when intercalated into DNA duplexes (Prince et al. 2001), thus increasing the $T_{\mathrm{m}} \mathrm{s}$ of the matched and mismatched duplexes. This effect is relatively greater for the mismatched structure, thus reducing the relative difference in observed peak $T_{\mathrm{m}}$. Nevertheless, these results show that for single-base variations, all three detection strategies give rise to $T_{\mathrm{m}}$ differences that enable unambiguous allele discrimination.

\section{Experiments With PCR-Amplified Targets}

To extend the model system findings, we also tested the iFRET, FRET, and intercalation dye systems on PCR-amplified targets. For these experiments we used the dynamic allelespecific hybridization (DASH) SNP-genotyping method (Howell et al. 1999). In brief, this method involves immobilization of a PCR-amplified target to a solid surface and subsequent annealing of a complementary oligonucleotide probe. The samples are then classified by generation and analysis of DNA-melting curves.

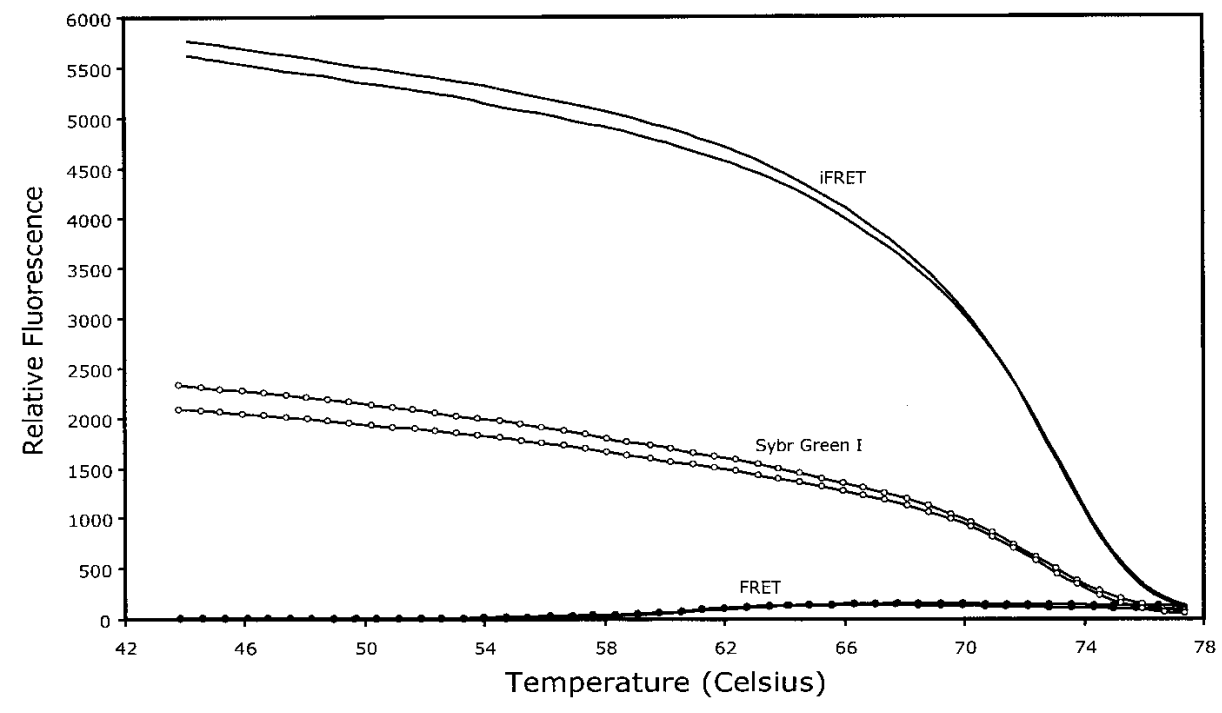

Figure 3 Comparision of signal intensity trends for Sybr Green I, Fluorescence resonance energy transfer (FRET), and induced (i)FRET. Melting curves are shown for the three indicated assay formats. Fluorescence measurements were recorded at the empirically determined emission maxima for respective dyes: for SYBR Green I detection: 541-545 nm, for FRET detection (FAM): 541-545 nm, and for iFRET detection (ROX): $611-615 \mathrm{~nm}$. 


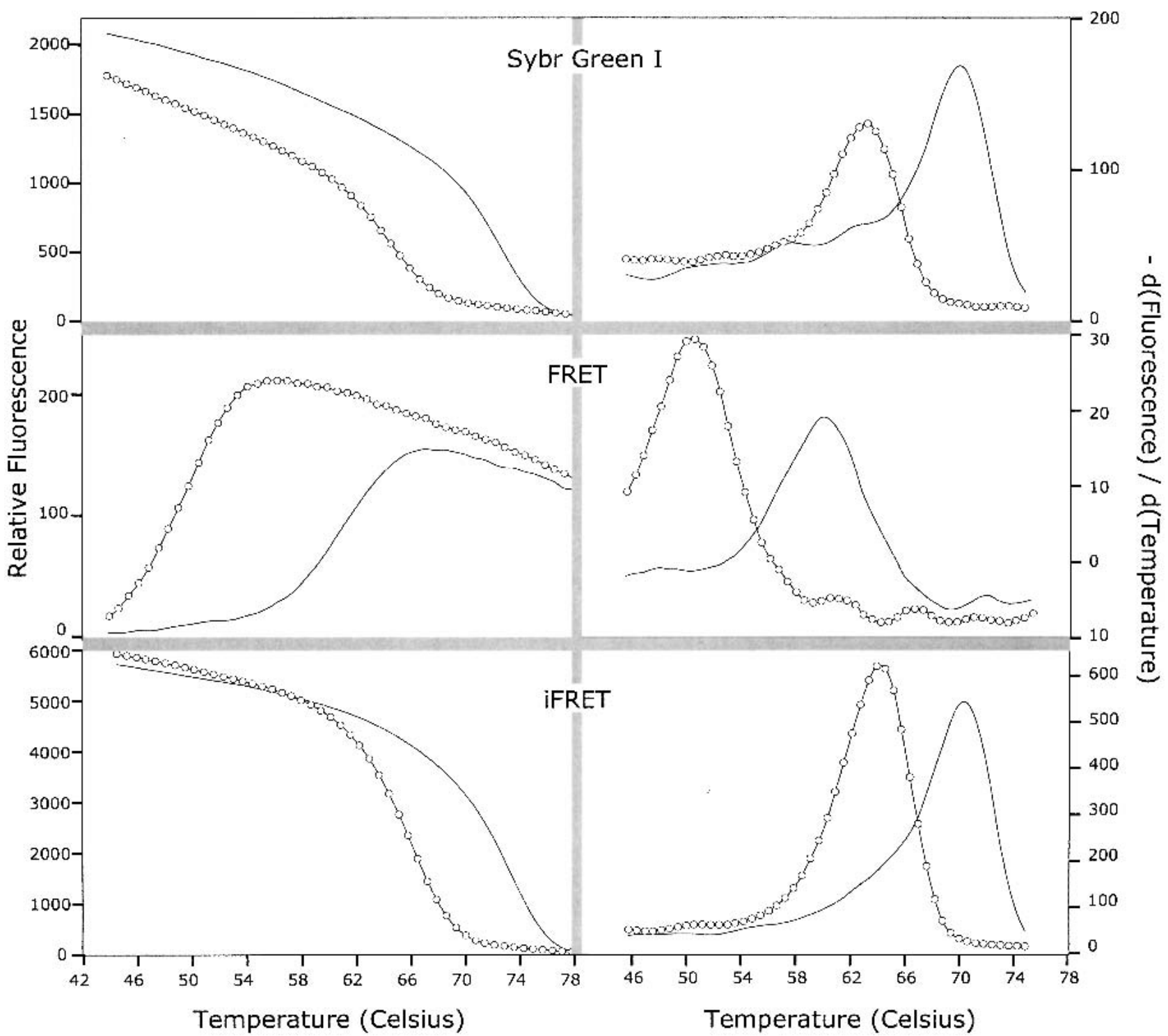

Figure 4 Melting curves for matched duplexes (solid lines) and single-base mismatch duplexes (hollow circles) are presented. For each indicated detection system, the graph to the left depicts fluorescence versus temperature, and the graph to the right is the derivative versus temperature.

For all three detection strategies, a 5'-biotin moiety was added to one of the two PCR primers to tag the 5 '-end of one strand of the PCR product and thereby enable its physical isolation. For FRET, a donor (FAM) group was also incorporated into the biotinylated PCR primer ( 4 bases from the 3 '-terminus) to ensure that the PCR product carried this moiety.

\section{Background Signals}

Rational predictions can be made about likely background fluorescence signals in the three systems. In theory and in practice (Prince et al. 2001), the use of SYBR Green I alone may generate background fluorescence due to interactions between the dye and regions of transient or permanent secondary structure in the immobilized target. In contrast, target molecule secondary structures alone should not give rise to this form of background signal with the FRET or iFRET alter- natives, because these schema require probe interactions to generate monitored fluorescence. Other background signals due to anomalous probe-target interactions can be readily avoided in all three systems simply by designing probe sequences to deliberately avoid unwanted sequence similarities with the target.

In our studies, these predictions were found to be true. This is illustrated by a DASH assay for an SNP (HGBASE ID: SNP000005045) involving the PCR primers 5'-biotinAGATACAGCACCAGCCTCAAGAC and 5'-GGCCTTCTC CCTGTAGATCCAC, and probe 5'-ACCCCCAGAACCGAC (the iFRET probe contained a 3'-ROX). The immobilized PCRamplified target creates secondary structure as observed by addition of SYBR Green I dye in the absence of any probe (Fig. $5 a)$. Background fluorescence from secondary structure is slightly more pronounced with the mismatch allele sequence (solid line) than the match allele target sequence (hollow

\section{Genome Research}




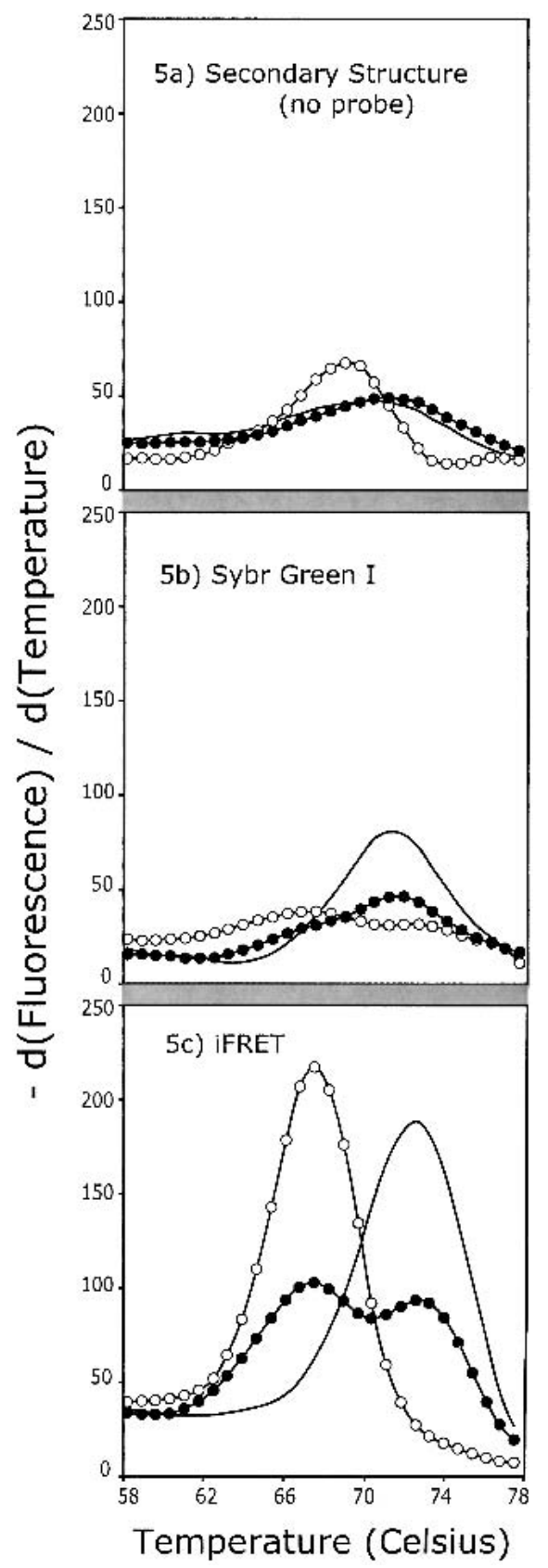

Figure 5 Melting curves of three DNA samples. Negative derivatives of DASH melting curves for PCR products for homozygous match (solid line), heterozygous (filled circles), and homozygous mismatched (hollow circles). (A) Secondary structure (no probe) signals are compared with $(B)$ probe/target duplex signals created by Sybr Green I and $(C)$ iFRET detection schemes.

circles). If unlabeled probe is included, the observed SYBR Green I dye fluorescence becomes a combination of background fluorescence together with fluorescence from the probe-target duplex (Fig. 5b). The denaturation signal (peak) of the matched and mismatched probe-target duplexes becomes so obscured that genotype calling by SYBR Green dye alone is made difficult for this SNP. However, when the iFRET alternative is tried (Fig. 5c), the procedure effectively isolates the true signals from the now nonexistent background, allowing the three distinct genotypes to be clearly differentiated.

A third form of background would be predicted to be intrinsic to the FRET system but completely avoided by the iFRET system. This background signal stems from the fact that with FRET, one is forced (by the intrinsic weakness of FRET acceptor signals) to measure donor fluorescence. Donor moieties will unavoidably create some background fluorescence whenever acceptor quenching is incomplete, either because of inefficiencies of the FRET chemistry or because no acceptor-carrying probe became hybridized to the target. In contrast, iFRET acceptor signals are strong enough to be monitored in place of the donor signals, and hence background donor fluorescence emission is completely irrelevant. Furthermore, for iFRET, not only should the acceptor stop fluorescing because of physical separation from the donor (dye) upon probe-target denaturation, but the dye-emitted energy that excites the acceptor will also be simultaneously extinguished. Hence, the iFRET system alone should be typified by virtually no irrelevant background fluorescence, and show the most marked changes in fluorescence with changes in DNA-duplex status. As shown in Figures 4 and 5, this is indeed the case. The iFRET curves are found to give consistently low background noise and the most distinct denaturation peaks of all three tested systems.

\section{DISCUSSION}

We have shown that iFRET, FRET, and SYBR Green I strategies can be successfully used for fluorescence generation applied to the detection of DNA hybridization and the generation of DNA-melting curves. All three approaches may also be used as a basis for the detection of single-base differences in the target molecules, as in the DASH genotyping procedure. Of the three alternatives, the newly developed iFRET system offers some significant advantages. A concise summary of pros and cons for the three detection approaches is given in Table 1 .

The major advantage of using SYBR Green I alone is its low cost. The use of unmodified oligonucleotide probes is cheap, and fluorescence signal strengths are moderately high. However, this format suffers a major drawback, in that background signals emanating from secondary structures in target molecules may obscure or be confused with real genotyping signals.

Advantages of the FRET system are the increased window of resolution between different allele $T_{\mathrm{m}} \mathrm{s}$, the generally reduced background, and the potential for spectralmultiplexing of assays by using various fluorophores in parallel. However, the fluorescence signals generated by FRET are extremely weak, and this could severely compromise the po-

Table 1. Advantages and Disadvantages of Detection Strategies Based upon SYBR Green I, FRET, and iFRET

\begin{tabular}{lccc}
\hline & SYBR Green I & FRET & iFRET \\
\hline DASH compatible & Yes & Yes & Yes \\
Cost & Low & High & Medium \\
Fluorescence signal & Medium & Low & High \\
$T_{m}$ peak separation & Medium & High & Medium \\
Multiplex potential & No & Yes & Yes \\
\hline
\end{tabular}


tential for signal detection in such multiplexed assays. Also, FRET as described here requires the physical attachment of both the donor and acceptor moieties to the interacting DNA molecules. The synthesis of two labeled oligonucleotides (with one labeled internally) can be very expensive.

Significantly, our results show that the innovation of combining energy transfer between a double-strand specific dye, such as SYBR Green I, and a DNA-affixed acceptor (here termed iFRET) is an attractive alternative for fluorescence generation. It provides fluorescence signals of greatly enhanced magnitude, implying many advantages. For example, smaller volume or weaker PCR reactions will be possible to assay, and less sophisticated imaging equipment will be needed for signal detection. It also reduces cost by removing the necessity for a physically attached donor on one of the interacting DNA molecules, yet it preserves the spectral-multiplexing potential afforded by FRET. Most notably, it eliminates essentially all forms of fluorescence background, engendering very clean assays with all the benefits that naturally follow from this, such as allowing throughputs to increase and automation to replace human intervention. Although DASH was used as a platform for iFRET testing in this paper, other methods based on melting curve analysis of duplex DNAs could also benefit from this powerful iFRET concept.

\section{METHODS}

\section{Probes and Primers}

All oligonucleotide probes and PCR primers were purchased from Interactiva.

\section{PCR Conditions}

For experiments involving a PCR-amplified target, primers were designed using the Oligo 5.0 software (Molecular Biology Insights). PCR reaction mixtures (total volume: $25 \mu \mathrm{L}$ ) consisted of $10 \mathrm{ng}$ of genomic DNA, two PCR primers ( 3 pmole of one primer bearing a 5'-biotin label, and 15 pmole of the unlabeled primer), 0.75 units of AmpliTaq Gold DNA Polymerase (PE Corp), 5\% dimethylsulfoxide, $1 \times$ AmpliTaq Gold buffer, $3 \mathrm{mM} \mathrm{MgCl}$, and $0.2 \mathrm{mM}$ each dNTP. Thermal cycling consisted of an initial enzyme activation step at $94^{\circ} \mathrm{C}$ for $10 \mathrm{~min}$, followed by 40 cycles of denaturation at $94^{\circ} \mathrm{C}$ for $15 \mathrm{sec}$ and at the appropriate annealing temperature for $30 \mathrm{sec}$. PCR was performed on a Touchdown temperature cycling apparatus (Thermo-Hybaid). After amplification, $5 \mu \mathrm{L}$ was taken from several random samples and examined by electrophoresis on a 3.0\% low-melt agarose gel for amplification quality control.

\section{Coupling of DNA Targets to a Microtiter Plate}

The immobilization procedure for model systems involved mixing 5 pmole of 5 '-biotinylated oligonucleotide target with $25 \mu \mathrm{L}$ of HEN buffer (0.1 M HEPES, $10 \mathrm{mM}$ EDTA, $50 \mathrm{mM}$ $\mathrm{NaCl}$ at $\mathrm{pH} 8.0$ ) and transferring the solution to a well of a streptavidin-coated microtiter plate (Thermo-Hybaid). The sample was left at room temperature for at least $30 \mathrm{~min}$ to allow affinity capture of the biotinylated targets to the streptavidin-coated surface. The solution was removed and the wells were rinsed once with $50 \mu \mathrm{L}$ of HEN.

For experiments using PCR-amplified targets, biotinylated PCR products (labeled at the $5^{\prime}$-end of one strand through one PCR primer carrying a $5^{\prime}$-biotin moiety) were diluted to $50 \mu \mathrm{L}$ by addition of $25 \mu \mathrm{L}$ of HEN buffer, and $25 \mu \mathrm{L}$ was transferred to a well of a streptavidin-coated microtiter plate. After a binding time of at least $30 \mathrm{~min}$ at room temperature, the solution was removed, and the wells were rinsed twice with $50 \mu \mathrm{L}$ of $0.1 \mathrm{M} \mathrm{NaOH}$. These rinses served to remove the nonbiotinylated strand, leaving only the biotinylated strand of the PCR product bound to the well surface. Then, the wells were rinsed once with $50 \mu \mathrm{L}$ of HEN buffer for neutralization.

\section{Hybridization of Allele-Specific Oligonucleotides}

For hybridization of allele-specific oligonucleotides, 15 pmole of the appropriate allele-specific oligonucleotide probe was added to reaction wells in $50 \mu \mathrm{L}$ of HEN. To assist probe hybridization, the plate was heated to $85^{\circ} \mathrm{C}$ on a dry, hot block (Techne) and allowed to cool to room temperature over 3-5 min. The hybridization solution was then discarded, and the wells were rinsed once in $50 \mu \mathrm{L}$ of HEN to remove any excess probe. Finally, a detection solution of $50 \mu \mathrm{L}$ of HEN was added. For testing the intercalation and iFRET strategies, the detection solution also contained a 1:10,000 dilution of SYBR Green I (Molecular Probes).

\section{Fluorescence Melting Curves}

Fluorescence measurements were produced and recorded using the ABI PRISM 7700 Sequence Detection System (Applied Biosystems). The software was programmed to heat the samples from $35^{\circ} \mathrm{C}$ to $85^{\circ} \mathrm{C}$ at $0.3^{\circ} \mathrm{C} / \mathrm{sec}$ while continuously monitoring fluorescence. The optimal emission frequency for each fluorescent dye was determined empirically. For the intercalation strategy, SYBR Green I fluorescence was measured at 541-545 $\mathrm{nm}$. For FRET, we measured FAM donor fluorescence at 541-545 nm. The alternative of measuring 6-carboxy-X-rhodamine (ROX) acceptor signals was not done because these second level signals were extremely weak. In contrast, for iFRET, the ROX acceptor fluorescence was very strong and able to be monitored effectively at 611-615 $\mathrm{nm}$. Plotting fluorescence versus temperature generated melting curves. Plotting the derivative of fluorescence versus temperature revealed melting temperature peaks that were more convenient for allele discrimination and genotype calling.

\section{ACKNOWLEDGMENTS}

We gratefully acknowledge the Carl Tryggers Foundation for Scientific Research and Thermo-Hybaid for funding that helped with aspects of our research undertakings. We also give thanks to the Center for Genomics and Bioinformatics (CGB) at Karolinska Institute and Pharmacia Corporation for provision of enabling infrastructure. The publication costs of this article were defrayed in part by payment of page charges. This article must therefore be hereby marked "advertisement" in accordance with 18 USC section 1734 solely to indicate this fact.

\section{REFERENCES}

Bernard, P.S., Lay, M.J., and Wittwer, C.T. 1998. Integrated amplification and detection of the C677T point mutation in the methylenetetrahydrofolate reductase gene by fluorescence resonance energy transfer and probe melting curves. Anal. Biochem. 255: 101-107.

Cardullo, R.A., Agrawal, S., Flores, C., Zamecnik, P.C., and Wolf, D.E. 1988. Detection of nucleic acid hybridization by nonradiative fluorescence resonance energy transfer. Proc. Natl. Acad. Sci. 85: 8790-8794.

Didenko, V.V. 2001. DNA probes using fluorescence resonance energy transfer (FRET): Designs and applications. Biotechniques 31: $1106-1120$.

Holland, P.M., Abramson, R.D., Watson, R., and Gelfand, D.H. 1991. Detection of specific polymerase chain reaction product by utilizing the 5'-3' exonuclease activity of Thermus aquaticus DNA polymerase. Proc. Natl. Acad. Sci. 88: 7276-7280. 
Howell, W.M., Jobs, M., Gyllensten, U., and Brookes, A.J. 1999. Dynamic allele-specific hybridization. A new method for scoring single nucleotide polymorphisms. Nat. Biotechnol. 17: 87-88.

Prince, J.A., Feuk, L., Howell, W.M., Jobs, M., Emahazion, T., Blennow, K., and Brookes, A.J. 2001. Robust and accurate single nucleotide polymorphism genotyping by dynamic allele-specific hybridization (DASH): Design criteria and assay validation. Genome Res. 11: 152-162.

Solinas, A., Brown, L.J., McKeen, C., Mellor, J.M., Nicol, J., Thelwell, N., and Brown, T. 2001. Duplex Scorpion primers in SNP analysis and FRET applications. Nucleic Acids Res. 29: E96.

Stryer, L. and Haugland, R.P. 1967. Energy transfer: A spectroscopic ruler. Proc. Natl. Acad. Sci. 58: 719-726.
Tyagi, S. and Kramer, F.R. 1996. Molecular beacons: Probes that fluoresce upon hybridization. Nat. Biotechnol. 14: 303-308.

$\mathrm{Wu}, \mathrm{P}$. and Brand, L. 1994. Resonance energy transfer: Methods and applications. Anal. Biochem. 218: 1-13.

\section{WEB SITE REFERENCES}

http://www.probes.com; homepage of Molecular Probes.

Received March 27, 2002; accepted in revised form July 17, 2002. 


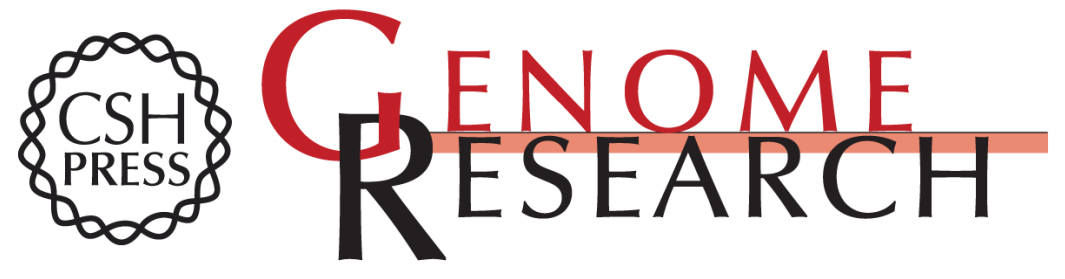

\section{iFRET: An Improved Fluorescence System for DNA-Melting Analysis}

W. Mathias Howell, Magnus Jobs and Anthony J. Brookes

Genome Res. 2002 12: 1401-1407

Access the most recent version at doi:10.1101/gr.297202

References This article cites 9 articles, 4 of which can be accessed free at:

http://genome.cshlp.org/content/12/9/1401.full.html\#ref-list-1

\section{License}

Email Alerting Receive free email alerts when new articles cite this article - sign up in the box at the Service top right corner of the article or click here.

\section{Affordable, Accurate Sequencing.}

To subscribe to Genome Research go to: https://genome.cshlp.org/subscriptions 